\title{
Formation of sharp edges and planar areas of asteroids by polyhedral abrasion
}

\author{
G. Domokos ${ }^{1,2}$, A. Á. Sipos ${ }^{1}$, Gy. M. Szabó ${ }^{4}$, and P. L. Várkonyi ${ }^{1}$
}

Received —

\footnotetext{
${ }^{1}$ Department of Mechanics, Materials, and Structures, Budapest University of Technology and Economics, Müegyetem rkp. 3, Budapest 1111, Hungary

${ }^{2}$ Trinity College, Cambridge, CB2 1TQ

${ }^{3}$ Department of Experimental Physics, University of Szeged, Dóm tér 9. Szeged 6720, Hungary
}

${ }^{6}$ E-mail: gd321@cam.ac.uk, szgy@titan.physx.u-szeged.hu 


\begin{abstract}
While the number of asteroids with known shapes has drastically increased over the past few years, little is known on the the time-evolution of shapes and the underlying physical processes. Here we propose an averaged abrasion model based on micro-collisons, accounting for asteroids not necessarily evolving toward regular spheroids, rather (depending on the fall-back rate of ejecta) following an alternative path, thus confirming photometry-derived features, e.g. existence of large, relatively flat areas separated by edges. We show that our model is realistic, since the bulk of the collisions falls into this category.
\end{abstract}

Subject headings: Solar system: minor planets, asteroids 


\section{Introduction}

The number of asteroids with known shapes has increased significantly in the past years. 8 asteroids were visited by fly-by missions, providing high-resolution images (e.g. Veverka et al. 1994, Chapman et al. 1995, Robinson et al. 1995, Veverka et al. 1999, Oberst et al. 2001, Duxbury et al. 2004, Saito et al. 2006, Schulz et al. 2008). Radar observations can also reveal the envelope of the shape (e.g. Ostro et al. 1988). A very promising tool of shape identification is the inversion of photometric light curves (Kaasalainen and Troppa, 2001, Kaasalainen et al 2002, KTP2002 hereafter), which resulted in photometric models for $\approx 100$ asteroids, so far. These new efforts and techniques lead to the rapid increase of the number of known asteroid shapes and we can expect this number to keep growing in the future.

These shapes have not been globally classified in the literature, but one can easily recognize some typical shape properties. On one hand, there are some asteroids with highly nonconvex shape (e.g. 3908 Nyx), there are bifurcated objects (Kleopatra), earthnut-like forms (Eros), all of which may be results of typical formation processes. On the other hand, several asteroids have a strikingly simple shape envelope with large flat surfaces bordered by relatively sharp edges. A significant portion of photometric shapes apparently share these features (e.g. 60531993 BW, Ďurech et al. 2002, 101151992 KS, Busch et al. 2005, 1580 Betulia, 1980 Tezcatlipoca, 2100 Rha-Shalom, Kaasalainen et al. 2004). These observations are often attributed to local concavities (e.g. Ďurech 2002) or albedo effects (KTP2002), i.e. they have been claimed to be artefacts of the photometric method. However, close-up images from spacecrafts also reveal that the presence of prominent flat areas and sharp edges is typical for at least half of the small asteroids (e.g. Ida, Gaspra, Annefrank, Steins). Recently, Troppa et al (2008) confirmed the presence of flat areas on photometric shapes and found that the number of flat areas/facets decreases with the 
elongation of the shape (or its convex hull). While photometric imaging certainly does not provide sufficient information to quantify the uncertainities associated with the mentioned qualitative geometric features, these images suggest that at least some asteroids may not evolve towards spheroids. Our goal is to explore this avenue by applying a simple averaged abrasion model.

Recently, several studies have been dedicated to explain the shapes and surface properties. The conclusions point to different evolution scenarios; they also help to better understand the relation between collisions and the global shape. The following basic scenarios were proposed:

- Formation of primordial shapes in disrupting, energetic collisions (Michel et al 2003), followed by continuous formation and erosion of craters (Housen and Holsapple, 2003, O’Brien et al. 2006).

- Rubble pile structure asteroids can get very elongated forms as a result of tidal effects (Bottke et al. 1999).

- Evolution of rubble piles with elongated shapes toward a spherical form by seismic shaking of collisions with moderate energy ( Korycansky and Asphaug, 2003, KA2003 hereafter, Szabó and Kiss, 2008)

- Continuous alteration of the surface by low-energy collisions and space weathering that form small craters (Lazzarin et al. 2006).

The widespread concept is that small impactors always smoothen the global shape by eroding sharp edges (A. Conrad, personal communication). The root of this concept is the obvious $O(3)$-symmetry of the problem: the impacts have uniform radial intensity and one would expect that abrasion in this environment converges to an $O(3)$-symmetric object (sphere). 
Low-energy impactors remove small pieces from the surface of the asteroid, which either fall back to the surface (captured by the gravitational field), or they escape. A model based exclusively on the first scenario (KA2003) predicted convergence to spheres (or oblate ellipsoids for rotating asteroids). In this paper, we adress the second one. In contrast to natural expectations, abrasion by small, escaping fragments breaks the $O(3)$-symmetry, and leads to the formation of large planar areas and sharp edges. The global outcome for most initial shapes are low-order convex polyhedra (i.e. convex shapes bordered by a few planes), similar to at least a considerable fraction of asteroids. We also present preliminary results for a more realistic model, combining the effects of escaping material and the redistribution of ejecta on the asteroid's surface. The reported features appear to preserved in a certain parameter-range.

\section{Description of the model}

Abrasion due to micro-impacts is a complex, stochastic process, in which minor, discrete pieces are removed from the asteroid's surface in small collisions. Our model is an averaged continuum approach where the effect of large numbers of small, discrete impactors is modeled by continuous abrasion of the surface. The first step towards the construction

of the partial diffeential equation (PDE) is determining the average rates of abrasion on an asteroid's surface under the simplest assumptions for the discrete impactors (e.g. uniform distribution for the direction).

Let the asteroid surface at time $t$ be given by the endpoints of a two-parameter family of vectors $\mathbf{x}(p, q, t)$. We assume that a large number of small particles (relative to the asteroid) move along random straight lines (in coordinates fixed to the asteroid) and collide with the asteroid; subsequently they escape together with small, broken portions of the asteroid (Ronca and Furlong 1979). In case of impact trajectories we ignore gravitational 
effects as well as spinning; both are negligible at high-speed impacts. (The role of gravity, however, can not be neglected when considering material falling back to the surface; the model we first discuss assumes that all material escapes, later in the paper we develop a general model including non complete ejecta escape.)

In particular, we assume constant and uniform (i.e. time- space- and directionindependent) radial intensity $\Phi$ of particle collisions (average number of collisions per unit time, unit area and unit solid angle), resulting, in case of convex surfaces, in constant and uniform surface intensity $m$ of particle collisions (average number of collisions per unit time, and unit surface area). We assume that the volume of material abraded from unit surface per unit time, or, equivalently, the abrasion speed $\partial \mathbf{x}(p, q, t) / \partial t$ in the direction of the inward directed surface normal $\mathbf{n}(p, q, t)$ is determined by the surface intensity $m$ and the abrasion "efficiency" of the impactors. The latter could be expressed as a function $f(\beta)$ of the impact angle $\beta(0<\beta<\pi / 2)$ between $\mathbf{n}$ and the direction of the incoming particle. Abrasion speed can be obtained by integrating $\Phi f(\beta)$ over the $H S$ half-sphere and considering that the number of the incoming particles depend on $\beta$,

$$
\begin{array}{r}
\frac{\partial \mathbf{x}(p, q, t)}{\partial t}=\int_{H S} \Phi \cos \beta f(\beta) d S \cdot \mathbf{n}(p, q, t) \\
=\Phi \int_{0}^{\pi / 2} f(\beta) \cos \beta \sin \beta d \beta \cdot \mathbf{n}(p, q, t)
\end{array}
$$

In case of $f(\beta)=1$, Eq. 1 yields $|\partial \mathbf{x} / \partial t|=\Phi=m$, however, for any other $f(\beta)$ we still obtain the simple PDE called Eikonal equation for the abrasion of convex surfaces due to uniform micro-impacts:

$$
\frac{\partial \mathbf{x}(p, q, t)}{\partial t}=\text { constant } \cdot \mathbf{n}(p, q, t)
$$

Of course, primordial asteroid shapes are often locally concave. In these regions equation (1) should be integrated over its zenith angle, which is only a part of the half-sphere. Thus, the surface intensity $m$ (and the abrasion speed) is lower for concave 
surface points than for typical (generic) points on the convex hull, and therefore concave parts tend to disappear. In the rest of the paper, we focus on the convex case.

After establishing the appropriate averaged continuum model, our next goal is to show the resulting stable polyhedral geometric patterns.

Solutions of (2) are known as wave-fronts (Arnold, 1986), and singularity theory describes their evolution, Figure 1 illustrates the propagation of a planar wave-front starting from a convex, smooth curve. One of the most relevant features is that initially smooth surfaces evolve structurally stable singularities (cf. Figure 1b) and self-intersections along edges(Figure 1c). In a physical abrasion process, the solution is limited to the domain free of self-intersections (which is a weak solution of the PDE, (Sethian, 1985)): the aforementioned self-intersecting edges and vertices appear on the surface of the physically relevant domain (Figure 1d). In three dimensions, the situation is analogous: edges and vertices appear in the solution for all generic (typical) smooth initial shapes (Figure 2a,b). Structural stability means in this context that the appearance of these geometric features can be expected for almost all initial surfaces. Another feature of this process is that polyhedra evolve to polyhedra, with the number of faces monotonically decreasing in time (Figure 2c). These features imply that this process produces shapes with flat areas separated by marked edges and vertices, almost regardless of the starting configuration. One can also provide a simple and complete list of final limit shapes (depending on the initial shape): tetrahedron, elongated cigar-shape or a flat disc (Várkonyi et al., 2008, Domokos et al, 2009.). We demonstrate two of these limits numerically (Figure 2). Needless to say, more sophisticated discrete models would predict the process more accurately in the quantitative sense, however, our compact PDE model captures the most essential qualitative features.

Previously, the evolution of asteroid shapes was modelled in two dimensions by Ronca and Furlong (1979) under similar assumptions, however, involving two misleading concepts: 
(i) rate of volume loss was associated with radial abrasion speed (toward the barycenter) rather than speed normal to the surface, and (ii) a false interpretation of zenith angle lead to nonconstant abrasion speeds on convex surfaces and also to inconsistent results. Their model also predicts surface singulaities, however, otherwise radically different morphological characters then ours.

\section{Simulations}

Eq. (2) is a Hamilton-Jacobi type PDE, which can be efficiently simulated by level-set methods (Osher and Fedkiw, 2002) and fast-marching methods (Sethian, 1999). Both methods handle well singularities (edges and vertices). In this paper, a MATLAB-based implementation of the level-set method (Mitchell, 2008) was used to compute the evolution of various initial shapes.

As reported from experiments e.g. by Cappacioni et al. (1984), Catullo et al (1984) and Ryan et al (2000), the average axis ratio of asteroids resulting from fragmentation of larger objects is $1 / 1.4 / 2$ with large scatter in both ratios. We used initial axis ratios even closer to the sphere to demonstrate that the emergence of elongated shapes and edges is independent of the initial shapes.

Figure 2 shows the evolution of three different random initial shapes of which the first two have been picked from relatively smooth surfaces. Observe emerging sharp edges and plane areas, analogous to the planar wave front in Figure 1. Also, observe the tetrahedral limit in the first case and the increasing prolateness leading to a 'cigar-shape' in the second case. The third series starts with a multi-faceted polyhedral shape (which could be interpreted as the convex hull of a more general shape). Here the number of faces is reduced in abrasion, implying the emergence of low-order polyhedra (and finally, a flat 
tetrahedron). The emerging prolateness for many initial shapes confirms the observation of Troppa et al. (2008). The figure also contains photometric shapes (Troppa et al 2002, KTP2002, Kaasalainen et al. 2004) showing visual resemblance to the intermediate stage of the simulated examples. We emphasize, however, that it was not our goal to reconstruct the exact history of any particular asteroid shape, we merely intended to illustrate the strong qualitative agreement between photometric shapes and the simulated geometries.

All asteroid images shown in Figure 2 are the result of photometric modeling providing only approximate information. We also tried to reconstruct the evolution of one asteroid for which the shape is known in detail. Asteroid 5535 Annefrank, visited by Stardust (Duxbury et al. 2004) apparently has two components, the major part is similar to a tetrahedron. Figure 3a shows a hypothetical time-series chosen by trial-and error from simulations of randomly perturbed spheres. One stage of the evolution is a rounded polyhedron, strongly resembling 5535 Annefrank (Fig 3c).

So far, all simulations were based on our extreme assumption that all abraded material escapes the gravitational field. To check the robustness of the described qualitative features we included in our PDE model the effect of ejecta falling back to the surface. We integrated the gravitational potential $U$ for the evolving shape and assumed that some fraction $p$ of ejecta resettles to the surface with intensity proportional to $e^{U / \bar{U}}$ which is a maximum entropy approximation of the fallback rate. Here, $\bar{U}$ denotes the average value of $U$ on the surface. All our previous computations correspond to the case $p=0$, whereas the case $p=1$ represents the scenario described in more detail by (KA2003). Detailed evaluation of this unified model is beyond the scope of the current paper, however we briefly comment on the emerging behavior: for $p=1$ the shapes converge to a sphere (which would be perturbed if we considered rotational effects); for smaller $p$, the final shape is a round disk with two smooth sides surrounded by a sharp edge; the flatness of the disk is decreasing function of 
$p$. Figure $3 \mathrm{~b}$ shows an example with $p=0.25$, illustrating another remarkable feature of the process: the initial behavior resembles the case $p=0$ (Figure 3a), and the emerging polyhedral shape remains visible for a long time before being transformed to a disk.

\section{Conclusions}

We showed in this paper that a simple continuum model, based solely on the assumptions that asteroids have

- numerous, high-speed collisions with

- relatively small impactors

- arriving uniformly randomly from all directions

accounts for the appearance of flat areas, separated by edges and vertices, which is observable in the available data. Photometric imaging certainly does not provide sufficient information to quantify the uncertainities associated with the mentioned qualitative geometric features. Based on our present model we can conclude that asteroids do not necessarily evolve preferentially toward regular spheroids, but may (depending on the fall-back rate of ejecta) follow an alternative path, thus confirming photometry-derived features. As far as we know, ours is the only scenario reproducing these features.

Our simple model does not include several effects which also influence the abrasion. (For example, collisions with larger objects have totally different geometric effects: vertices and edges will be worn and appear less pronounced, craters are created, etc.). The global effects listed in Sec. 1 also play an important role in forming the final geometry. Quantitative predictions of shape evolution could only be achieved by more sophisticated, 
discrete models. After including the effect of gravity, as long as the majority of particles escape, edges remain a visible geometric feature.

The relevance of our model relies on the assumption that high-speed collisions with small impactors dominate the abrasion process. This is quite plausible, since the size distribution of asteroids (e.g. Tedesco et al. 2005, Parker et al. 2008) in the $>500 \mathrm{~m}$ range was found to be a quickly decreasing function following a power law of exponent $-2.5 \ldots-3$ The size distribution of smaller particles has not been examined directly, however numerical models for impact evolution predict even steeper (up to the -7 power) size distribution functions especially for small sizes (Michel et al. 2004). While it is beyond the scope of the current paper, it is possible to estimate an order of magnitude for the number of collisions needed to model the body (KA2003).

Abrasion processes governed by equation (2) occur also on much smaller scale: pebbles shaped by wind-blown sand, also known as ventifacts, evolve in a similar manner (Várkonyi et al., 2008, Domokos et al., 2009), the details of which process we are currently investigating.

Supports from OTKA grant T72146 (GD, AS, PV), OTKA K 76816 (GyMSz) and the "Bolyai János" Research Fellowship of the Hungarian Academy of Sciences (GyMSz) are gratefully acknowledged. The comments by the anonymous Referee greatly improved this paper. 

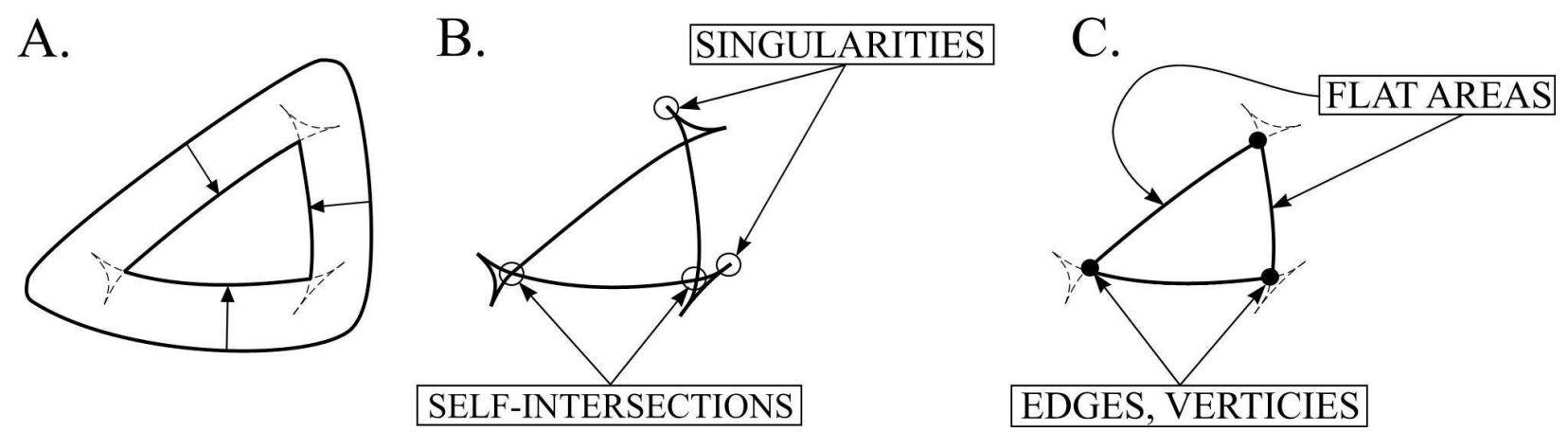

Fig. 1. - Propagation of wave front inside planar curve. 1a: Wave front before and after self intersection 1b: Generic (typical) singularities and self-intersections, occurring for almost all initial curves 1c :Physically relevant part of wave front, with edges resulting from selfintersections. 


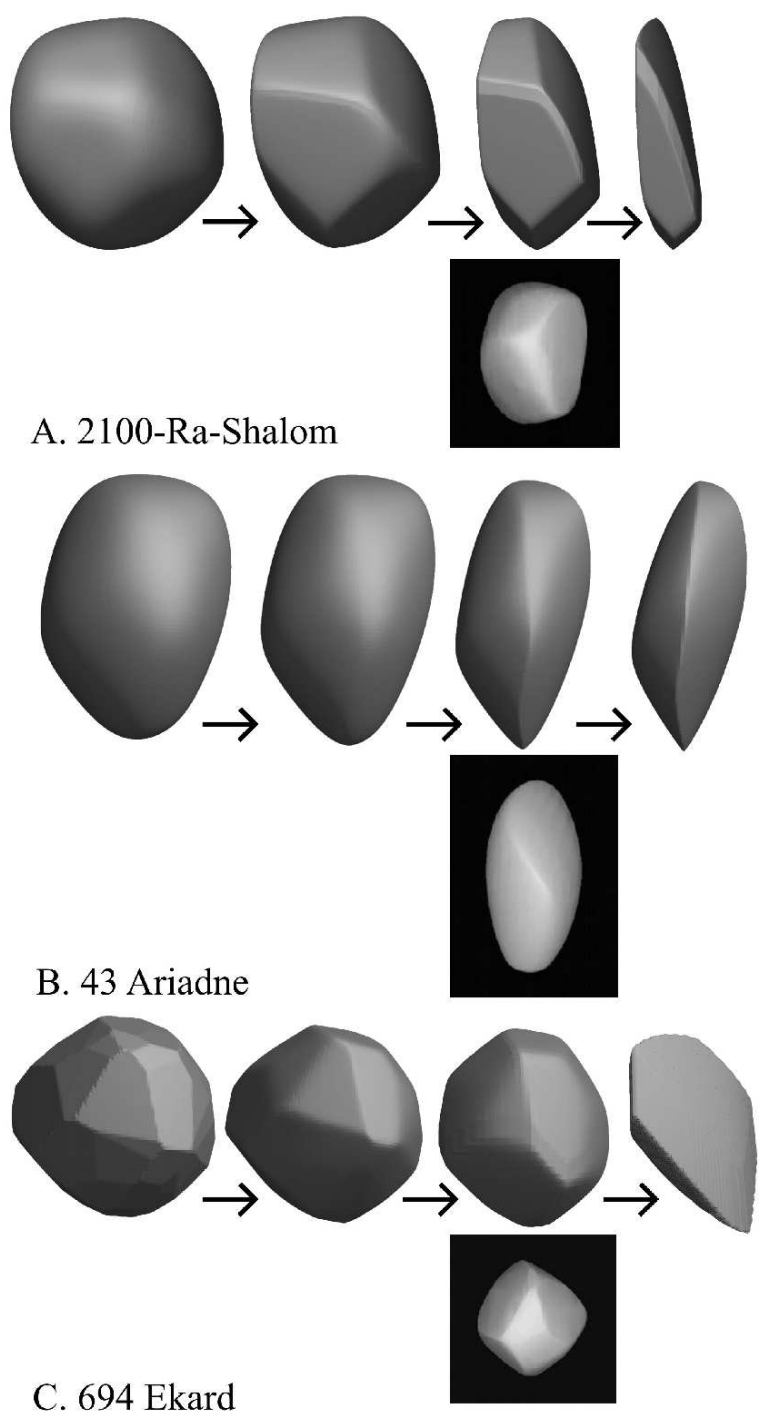

Fig. 2.- Some results of simulated shape evolution by abrasion. The examples show 2A: elongated polyhedron, compared to asteroid 2100 Ra-Shalom (Kaasalainen et al. 2004) 2B: flat disk, compared to asteroid 43 Ariadne (KTP2002) 2C: polyhedron, compared to asetroid 694 Ekard (Troppa et al. 2004) Photometric shape models are shown just to indicate qualitative agreement. 


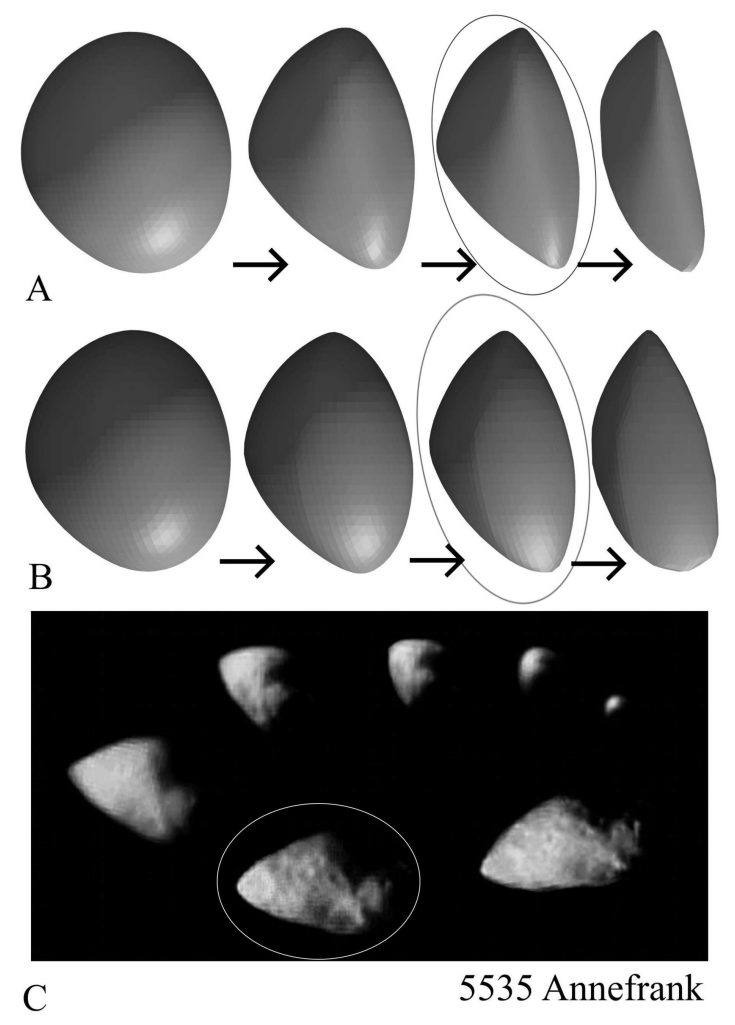

Fig. 3.- Simulation for Asteroid 5535 Annefrank. 3A: Hypothetical evolution of the shape under the modeled abrasion process. In phase 3 the shape shows remarkable similarity to 5535 Annefrank. 3B: Hypothetical evolution of the shape under the unified model, with $25 \%$ of particles captured by gravity. 3C: Asteroid 5535 Annefrank as seen from the Stardust spacecraft (small images) 


\section{REFERENCES}

Arnold, V.I., 1986, Catastrophe Theory, Springer, Berlin (2nd edition)

Botke, W. F. Jr., Richardson, D. C., Michel, P. \& Love, S. G., 1999, AJ, 117, 1921

Busch, M. W. et al., 2005, Icarus, 181, 145

Cappacioni, F et al., 1984, Nature, 308832

Catullo, V., Zappala, V., Farinella, P. \& Paolicchi, P., 1984, A\&A, 138, 4464

Chapman, C. et al., 1995, Nature, 374, 783

Domokos, G. et al., 2009, Periodica Polytechnica, Arch. 40, 1.

Ďurech, J, 2002, Icarus, 159, 192

Duxbury, T. C. et al., 2004, J. Geophysical Research, 109, 2

Housen, K. R. \& Holsapple, K. A., 2003, Icarus, 163, 102

Kaasalainen, M., Troppa, J. \& Piironen, J. 2002, Icarus, 159, 369 (KTP2002)

Kaasalainen et al., 2004, Icarus, 167, 178

Korycansky and Asphaug 2003, Icarus 163, 374

Lazzarin, M et al., 2006, ApJ, 647, 179

Michel, P., Benz, W. \& Richardson, D. C., 2003, Nature, 421, 608

Michel, P. et al., P\&SS, 52

Mitchell, I. M., 2008, The Flexible, Extensible and Efficient Toolbox of Level Set Methods, J. Scientific Computing, 35, 300-329. 
Oberst, J. et al., 2001, Icarus, 153, 160

O'Brien, D. P., Greenberg, R. \& Richardson, J. E., 2006, Icarus, 183, 79

Osher, S., Fedkiw, R., 2002, Level Set Methods and Dynamic Implicit Surfaces. Springer-Verlag.

Ostro, S. J., Connelly, R. \& Belkora, L., 1988, Icarus, 73, 150

Robinson, M. et al., 1995, Meteoritics, 30, 566

Ronca, L.B. \& Furlong, R.B, 1979, EMP, 21, 409

Ryan, E.V., Ann. Rev. P\&SS, 28, 367

Saito, J. et al., 2006, Science, 312, 1341

Schulz, R. et al., 2008, DPS, 40, 2829

Sethian, J.A. 1999, Level Set Methods and Fast Marching Methods, Cambridge University Press.

Sethian, J.A., 1985, Comm. Math. Phys., 101, 487.

Szabó, Gy.M. \& Kiss, L. L., 2008, Icarus, 196, 135

Troppa, J., et al., 2008, Icarus, 198, 91

Troppa, J. et al., 2003, Icarus, 164, 346

Veverka J., Belton, M., Klassen, K. \& Chapman, C., 1994, Icarus, 107, 2

Veverka J. et al, 1999, Icarus, 140, 3 
Várkonyi P. L., Domokos G. \& Sipos A. Á. 2008, Proc. of the $1^{\text {st }}$ Workshop on Mathematical Geosciences, June 11-12 2008, Freiberg, Germany. Available at: http://www.iamg.tu-freiberg.de/assets/docs/pdf/ProceedingsMatGeoS08/Peter Varkonyi.pdf 